Check for updates

Cite this: Nanoscale Adv., 2020, 2, 199

Received 25th October 2019

Accepted 14th December 2019

DOI: $10.1039 / c 9 n a 00679 f$

rsc.li/nanoscale-advances

\section{Diatom frustules enhancing the efficiency of gel polymer electrolyte based dye-sensitized solar cells with multilayer photoelectrodes}

\author{
T. M. W. J. Bandara, (D) *ab M. Furlani, ${ }^{C}$ I. Albinsson, ${ }^{c}$ Angela Wulff ${ }^{d}$ and B.-E. Mellander ${ }^{a}$
}

The incorporation of nanostructures that improve light scattering and dye adsorption has been suggested for dye-sensitized solar cells (DSSCs), but the manufacture of photonic and nanostructured materials with the desired properties is not an easy task. In nature, however, the process of light-harvesting for photosynthesis has, in some cases, evolved structures with remarkable wavelength-sensitive light-trapping properties. The present work is focused on enhancing the efficiency of quasi solid-state DSSCs by capitalizing on the light trapping properties of diatom frustules since they provide complex 3-dimensional structures for scattering and trapping light. This study reports a promising approach to prepare $\mathrm{TiO}_{2}$ nanocrystal $(14 \mathrm{~nm}$ ) based photo-electrodes by utilizing the waveguiding and photon localization effects of nanostructured diatom frustules for enhancing light harvesting without deteriorating the electron conduction. Single and double-layered photo-electrodes were prepared with different frustule/nanocrystal combinations and conformations on transparent conductive oxide substrates. This study clearly reports impressive efficiency and short circuit current density enhancements of about $35 \%$ and $39 \%$, respectively, due to the incorporation of diatom frustules extracted from a ubiquitous species. The SEM images obtained in this work reveal that the produced thin films had a remarkable surface coverage of evenly distributed frustules within the $\mathrm{TiO}_{2}$ nanoparticle layer. To the best of our knowledge, this study reports the first quasi solid-state DSSC based on a photo-electrode with incorporated bio-formed nanostructures.

\section{Introduction}

Dye-sensitized solar cells (DSSCs) have aroused growing interest due to the prospects of low cost and "greener fabrication"1 and also due to other factors such as their high efficiency under low

\footnotetext{
${ }^{a}$ Department of Physics, Chalmers University of Technology, Gothenburg, Sweden. E-mail: awijendr@yahoo.com

${ }^{b}$ Department of Physics, Postgraduate Institute of Science, University of Peradeniya, Sri Lanka

${ }^{c}$ Department of Physics, University of Gothenburg, Gothenburg, Sweden

${ }^{d}$ Department of Biological and Environmental Sciences, University of Gothenburg, Gothenburg, Sweden
}

light ambient conditions. ${ }^{2}$ Some improvements in performance have recently been achieved for DSSCs with the introduction of inorganic and organic nanostructured materials that offer a waveguiding effect and efficient electron transport in the photo-electrode..$^{3-5}$ Consequently, in order to improve the lightharvesting efficiency of DSSCs, the development of threedimensional photo-electrodes for enhancing effective dye adsorption, by improving the scattering of light within the electrode, guiding and trapping light and at the same time improving electron transport is a novel approach., ${ }^{3,5-7}$

The light-harvesting efficiency of DSSCs primarily depends on two factors:

(1) The surface density of dye molecules that photons can reach controls the number of photo-generated electrons since the dye is the light-absorbing species. Therefore, the effective surface area of the photo-electrode where the dye is adsorbed should be as high as possible to obtain the highest efficiency. In relation to this, the light should be scattered effectively within the nanostructured wide band-gap semiconductor layer to reach the dye molecules, avoiding self-shadowing effects.

(2) The photo-generated electrons should be transported to the external circuit efficiently before they recombine. Therefore, the photo-electrode should provide efficient charge transport in order to conduct photo-generated electrons to the external circuit.

Conventional high-efficiency photo-electrodes of DSSCs consist of a rather thin film of $\mathrm{TiO}_{2}$ nanoparticles that has a large surface area, covered by a monolayer of dye molecules to harvest sunlight. ${ }^{\mathbf{8 - 1 0}}$ Such $\mathrm{TiO}_{2}$ nanoparticle-based films are transparent with poor light scattering due to the small size of the particles, and this can result in limited light-harvesting efficiency. ${ }^{9-11}$

The light-harvesting ability of the $\mathrm{TiO}_{2}$ electrodes can thus be improved by increasing the available surface for dye loading and the effective light scattering by integration of various nanostructures into the photo-electrode. ${ }^{3,12-14}$ Conversely, the advantages offered by the relatively high surface area exhibited by various nanostructured films can be compromised by less 
efficient charge collection by the electrode. $\mathrm{TiO}_{2}$ nano-wire or nano-rod based DSSCs have been studied in order to improve the electron transport; however, such cells have shown lower efficiencies compared to traditional nanoparticle-based cells, ${ }^{\mathbf{8}, 10,15,16}$ despite the improved electron transport. ${ }^{\mathbf{1 2 , 1 4}}$ The efficiency drop is likely due to the relatively small surface area of the modified electrodes that the dye could adsorb on, compared to that of conventional $\mathrm{TiO}_{2}$ nanoparticle-based films without such additives. Thus, the effective surface area is a crucial factor that governs the efficiency of DSSCs. Consequently, in order to enhance the energy conversion efficiencies, optical fibers have also been studied for use in DSSCs with the intention of improving the effective surface area of the $\mathrm{TiO}_{2}$ nanoparticle films, by capturing the wave-guiding effects. ${ }^{17,18}$

Design and fabrication of the desired nanostructures to scatter and trap light efficiently is, however, not an easy task. Nano-scale photonic light-trapping technologies and advanced nanostructures have been developed using sophisticated methods and costly instrumentation; ${ }^{19-22}$ however, reproducibility of the structure and morphology is problematic for

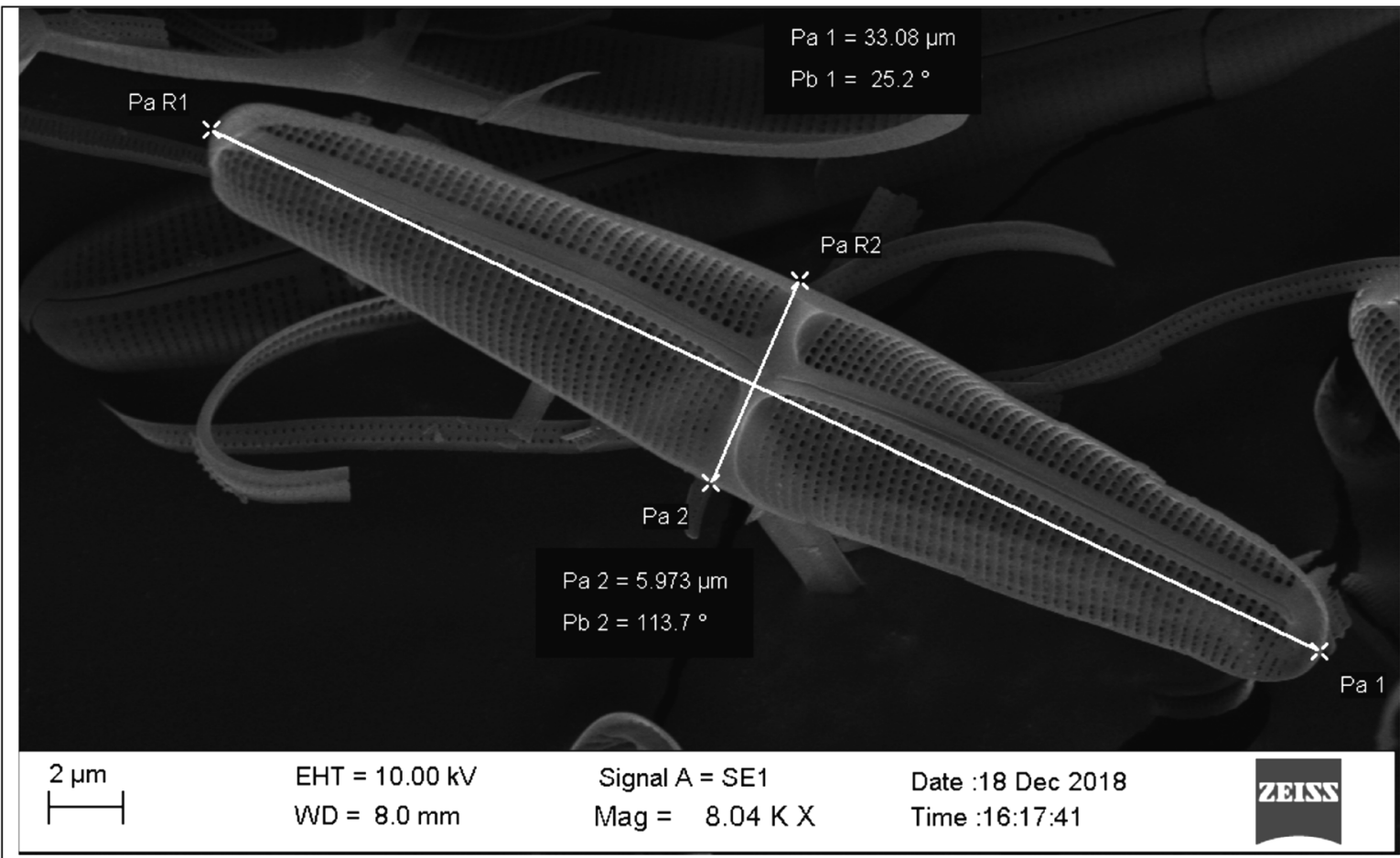

(a)
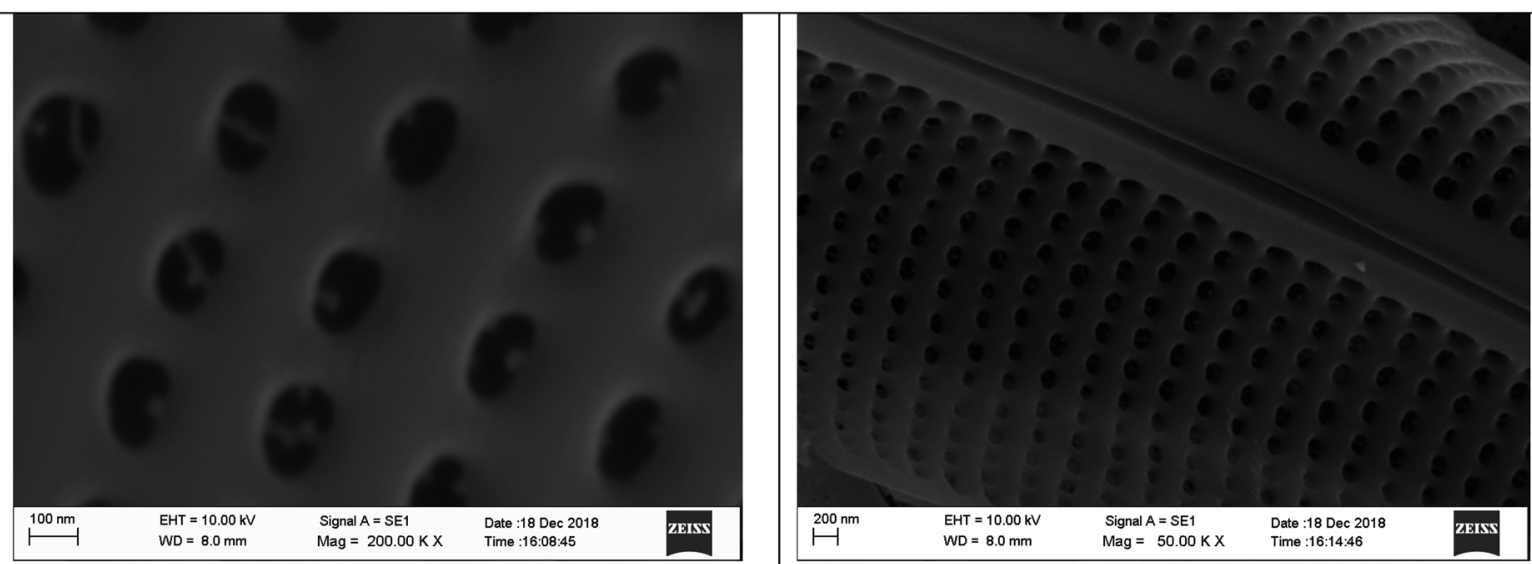

(b)

(c)

Fig. 1 SEM images of nanostructured diatom frustules extracted by the Swedish Algae Factory (Swedishalgaefactory.com) at (a) 8040 , (b) 50000 and (c) 200000 magnification. 
artificial nanostructures. In addition, mass-scale production of such structures can be very costly. However, considering an alternative approach, one could observe that nature has designed some structures able to harvest sunlight with remarkable light-trapping properties. ${ }^{2324}$ For example, in the process of photosynthesis, aquatic organisms produce organic compounds from $\mathrm{CO}_{2}$ by utilizing sunlight efficiently and effectively with the help of built-in silica nanostructures. ${ }^{24,25}$

Diatoms are abundant unicellular microscopic algae commonly found in all aquatic habitats on Earth. ${ }^{24,26,27}$ They are enclosed by a cell wall, named the frustule, made of silica. ${ }^{28,29}$ The role of the frustule is manifold; it provides mechanical stability, is a filter for nutrients, gases and water, contributes to the defense against viruses and harmful bacteria, and manipulates incoming light. The size of the frustules varies from $1 \mu \mathrm{m}$ to $1 \mathrm{~mm} ;{ }^{30,31}$ they have species-specific nanoscale patterns and shapes which have been hypothesized to be designed to provide efficient harvesting of visible light while providing physical protection for the cell. ${ }^{32-34}$ A variety of forms of diatoms are present in nature, and they are broadly divided into centric and pennate forms, depending on their symmetry. The pennate diatom frustules investigated in this study, processed and purified from a diatom monoculture by the Swedish Algae Factory, are shown in Fig. 1 at different magnifications. These structures have naturally evolved over millions of years and seem to be optimized for scattering light for the photosynthesis process. ${ }^{24}$ The studies performed by Chen et al. ${ }^{24}$ have shown through experiments and simulations that placing diatom frustules on the surface of light-absorption materials strongly enhances the optical absorption of visible light. Frustules are, therefore, ideal materials that can be used to increase lightharvesting in DSSCs. In addition, diatoms are widely available in aquatic habitats and frustules are extracted at low cost even on a mass scale. ${ }^{35}$

Some investigations into the incorporation of diatom frustules in the photo-electrode of DSSCs have been performed lately. An appreciable study was performed by Toster et al. ${ }^{\mathbf{3 4}}$ showing an improvement of the energy conversion efficiency from $3.5 \%$ to $4.6 \%$ by incorporating diatom frustules; however, they used conventional liquid electrolytes. In addition, Campbell et $a l .{ }^{36}$ reported improved solar cell efficiencies from 1.68 to 1.82 and further to $1.91 \%$, again using a volatile solvent electrolyte. Huang et $a l .{ }^{37}$ reported that the power conversion efficiency improved from $3.81 \%$ to $5.26 \%$ using frustule containing $\mathrm{TiO}_{2}$ paste instead of conventional $\mathrm{TiO}_{2}$ paste for photoelectrode preparation. These authors have thus obtained efficiencies over $5 \%$ for the first time in solar cells incorporated with diatom frustules. However, the physical and chemical stability of these cells can suffer due to common issues that originate from the use of liquid electrolytes. Therefore, in the present study, the liquid electrolyte in the cell is replaced by an appropriate gel polymer electrolyte, and the use of volatile solvents for the electrolyte preparation is avoided.

Even though the incorporation of frustules has led to relatively large improvements, the efficiencies are still low in these cells compared to those of conventional high-efficiency DSSCs assembled using thin films of $\mathrm{TiO}_{2}$ nanoparticles, which show efficiencies above $12 \% .^{8-10,38,39}$ Besides, the use of a volatile liquid electrolyte hinders the practical applications of DSSCs as an outdoor device. Therefore, the present work is focused on enhancing the efficiency of quasi solid-state DSSCs capitalizing on the light trapping properties of diatom frustules since the diatom frustule provides an inexpensive way for accessing complex 3-dimensional structures designed in nature with light scattering and trapping properties. For cell assembly, gel polymer electrolytes were selected since they have been proven to offer higher stability and easier handling compared to liquid electrolytes. ${ }^{40}$ To the best of our knowledge, the present work reports the first quasi solid-state DSSC based on a photoelectrode with incorporated bio-formed nanostructures.

\section{Experimental}

\subsection{Photo-electrode preparation}

2.1.1 Materials. The frustules, purified at the Swedish Algae Factory - Sweden (batch K632-freeze-dried), were mixed with $\mathrm{TiO}_{2}$ nanoparticles in $0.1 \mathrm{M} \mathrm{HNO}_{3}$, forming a slurry, and layered in various combinations and conformations on glass substrates with a transparent conductive oxide film of fluorine-doped tin oxide (FTO) (sheet resistance $7 \mathrm{ohm} /$ square), purchased from Solaronix, Switzerland. The photo-electrodes were prepared with single and double-layered films using $\mathrm{TiO}_{2}$ nanoparticles (received from Evonik, Germany) of particle size 14 nm (P90). These multi-layer films were then stained with N719 dye and used as the photo-anodes in photoelectrochemical solar cells.

\subsubsection{Layer preparation}

2.1.2.1 Titania layer without frustules. The titania layer without frustule incorporation was prepared from a $\mathrm{TiO}_{2}$ slurry obtained by mixing and grinding a mixture of $0.5 \mathrm{~g}$ of $\mathrm{P} 90$ powder and $2 \mathrm{ml}$ of $0.1 \mathrm{M} \mathrm{HNO}_{3}$ in an agate mortar for about $20 \mathrm{~min}$. Then this slurry was spin-coated at $1000 \mathrm{RPM}$ on well cleaned FTO glass substrates or on pre-prepared $\mathrm{TiO}_{2}$ substrates.

2.1.2.2 Titania layer with frustules. A mixture prepared using $\mathrm{TiO}_{2}$ P90 powder and diatom frustules was spin-coated at 1000 RPM. For this purpose, $\mathrm{TiO}_{2} \mathrm{P90}$ powder and diatom frustules were mixed according to a weight ratio of $\mathrm{P} 90$ : frustules = $100: 1$ ( $0.5 \mathrm{~g}$ of P90 powder and $0.005 \mathrm{~g}$ frustules in $2 \mathrm{ml}$ of $0.1 \mathrm{M} \mathrm{HNO}_{3}$ ). The slurry was homogenized by sonicating the mixture for about $2 \mathrm{~h}$ at room temperature. Before spin coating, the mixture was allowed to rest at room temperature for $12 \mathrm{~h}$ to allow diffusion of the $\mathrm{TiO}_{2}$ nanoparticles into the pores in the frustules.

After spin coating, each layer was air-dried for about $24 \mathrm{~h}$ and then calcined at $450{ }^{\circ} \mathrm{C}$ for about $2 \mathrm{~h}$. Heating and cooling were conducted at $1.5{ }^{\circ} \mathrm{C} \min ^{-1}$ and $-1.5{ }^{\circ} \mathrm{C} \min ^{-1}$ rates, respectively. The air drying and calcination procedures were repeated for each and every layer. The above-mentioned layers were fabricated in different configurations to prepare the photoelectrodes.

2.1.3 Dye sensitization. The $\mathrm{TiO}_{2}$ films containing single or multiple layers were photo-sensitized using N719 dye for use as photo-anodes. For this purpose, the pre-prepared single or multi-layered $\mathrm{TiO}_{2}$ photo-electrodes were immersed in 
a saturated ethanol solution of di-tetrabutylammonium cisbis(isothiocyanato)bis(2,2'-bipyridyl-4,4'-dicarboxylato)ruthenium(II) dye (N719). When the electrodes were dipped in the dye solution, the temperature of the dye solution and the electrode was initially maintained at approximately $60{ }^{\circ} \mathrm{C}$ and was then allowed to decrease to room temperature. The electrodes were kept for $24 \mathrm{~h}$ in the solution to complete the dye adsorption. Finally, the dye molecules loosely bound to the electrode were washed away by rinsing the films with ethanol. The dye adsorbed electrodes were then used as photo-anodes for the DSSC assembly.

\subsection{Electrolyte preparation}

The electrolyte sample was prepared by using polyacrylonitrile (PAN) $\left(M_{\mathrm{w}} 150000\right)(0.100 \mathrm{~g})$, ethylene carbonate $(0.417 \mathrm{~g})$, propylene carbonate (0.385 g), 4-tert-butylpyridine (4TBP) (0.022 $\mathrm{g})$, rubidium iodide (RbI) (0.048 g), tetrahexylammonium iodide $\left(\mathrm{Hex}_{4} \mathrm{NI}\right)$ (0.045 g), and 1-butyl-3-methylimidazolium iodide (BMII) $(0.0126 \mathrm{~g})$. The molar ratio of the components in the gel polymer electrolyte was PAN : EC : PC : BMII : $\operatorname{Hex}_{4} \mathrm{NI}: \mathrm{RbI}: 4 \mathrm{TBP}: \mathrm{I}_{2}=$ $10: 25: 20: 0.25: 0.5: 1.2: 0.85: 0.19$. Therefore, the stoichiometric composition can be written as $(\mathrm{PAN})_{10}(\mathrm{EC})_{25}(\mathrm{PC})_{20}(-$ BMII $)_{0.25}\left(\mathrm{Hex}_{4} \mathrm{NI}\right)_{0.5}(\mathrm{RbI})_{1.2}\left(\mathrm{I}_{2}\right)_{0.19}$. The weight composition of the electrolyte is given in Table 1.

For sample preparation, EC, PC and 4TBP were mixed in a closed glass bottle under continuous stirring. The relevant amount of iodides, RbI, $\mathrm{Hex}_{4} \mathrm{NI}$, and BMII, was added to the mixture and stirred for a few more hours, and then $0.1 \mathrm{~g}$ of PAN was added to the salt solution and stirred further to obtain a homogeneous mixture. ${ }^{41}$ The resulting mixture was heated to $\sim 100{ }^{\circ} \mathrm{C}$ under continuous stirring until the mixture became a homogeneous and transparent slurry. The mixture was allowed to cool down under continuous stirring. When the temperature dropped to about $50{ }^{\circ} \mathrm{C}, 9.0 \mathrm{mg}$ of iodine $\left(\mathrm{I}_{2}\right)$ chips was also added to the slurry. The stirring was continued until the slurry cooled down to room temperature. The resulting gel polymer electrolyte was used to assemble the DSSCs.

\subsection{Solar cell assembly}

The DSSC was assembled by sandwiching a thin layer of the gel polymer electrolyte between the photo-electrode and the platinum-coated glass plate (counter electrode) by clipping them together.

Table 1 Composition of the gel polymer electrolyte

\begin{tabular}{llc}
\hline Component & Weight/g & Molar ratio \\
\hline PAN & 0.1000 & 10.00 \\
EC & 0.4175 & 25.00 \\
PC & 0.3849 & 20.00 \\
RbI & 0.0481 & 1.20 \\
$\mathrm{He}_{4} \mathrm{NI}$ & 0.0454 & 0.50 \\
$\mathrm{BMII}$ & 0.0126 & 0.25 \\
$4 \mathrm{TBP}$ & 0.0217 & 0.85 \\
$\mathrm{I}_{2}$ & 0.0090 & 0.19
\end{tabular}

\subsection{Solar cell measurements}

The fabricated solar cells were illuminated using a LOT-Oriel GmbH solar simulator, $1.5 \mathrm{AM}, 1000 \mathrm{~W} \mathrm{~m}^{-2}$ (one sun), in order to obtain the $I-V$ characteristics using an eDAQ Potentiostat and e-corder. The area of the cell exposed to light was 12 $\mathrm{mm}^{2}$, and the potential scan rate was $100 \mathrm{~V} \mathrm{~s}^{-1}$. Measurement scanning was performed in the direction from forward bias $(0.8$ V) to reverse bias $(-0.8 \mathrm{~V})$.

\subsection{SEM images}

The SEM pictures were captured with an Ultra 55 Leo at $10^{-5}$ mbar and $3 \mathrm{kV}$ inserting the samples flat (typically 3 at a time) on the sample holder, with no special treatment. SEM images of bare frustules were obtained, after coating gold on them, with a ZEISS EVO LS15 setup. The thickness of the layers has, where possible, been evaluated at the edge of the layer focusing on different surfaces and measuring the focus position.

\section{Results and discussion}

\subsection{DSSCs with a single layer photoelectrode without frustules}

The current density versus cell potential $(J-V)$ curves of the quasi solid-state DSSC prepared with a single-layer photo-electrode without frustules and a gel electrolyte based on PAN as the host polymer containing the binary salts $\mathrm{RbI}$ and $\mathrm{Hex}_{4} \mathrm{NI}$ are shown in Fig. 2. Measurements were conducted for about $3 \mathrm{~h}$ under continuous illumination of $1000 \mathrm{~W} \mathrm{~cm}^{-2}$ in order to check the short-term stability of the DSSC in addition to the cell performance. As shown in Fig. 2, the current density $\left(J_{\mathrm{sc}}\right)$ of the cell increases with time, from 6.2 to $7.0 \mathrm{~mA} \mathrm{~cm}{ }^{-2}$ within 3 hours under continuous irradiation. This increase can be a result of heating effects caused by prolonged irradiation of the DSSC since, in general, the charge transport kinetics increase with

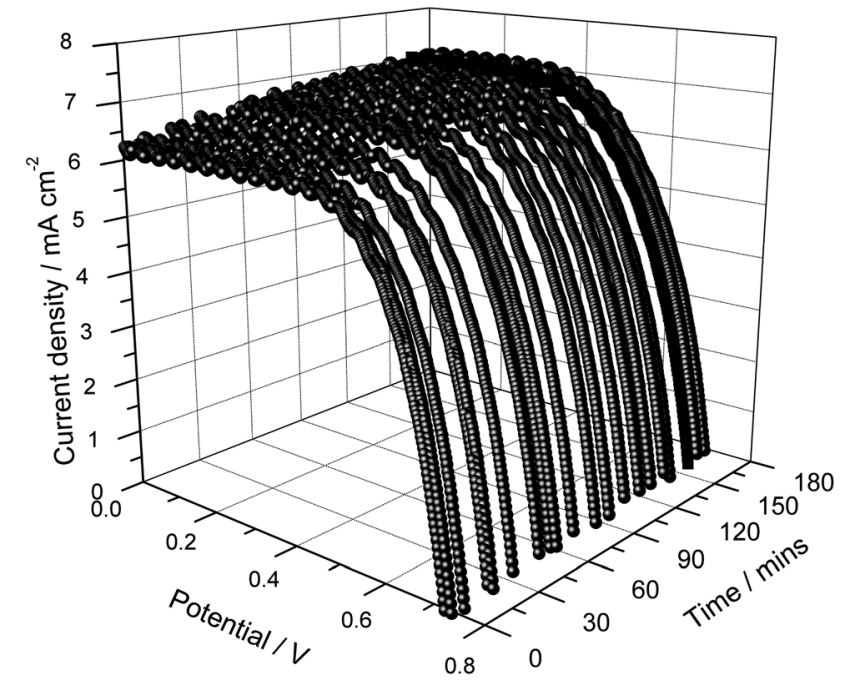

Fig. 2 Current density versus cell potential as a function of illumination time of the DSSC assembled using a single layer photo-electrode without frustules. 


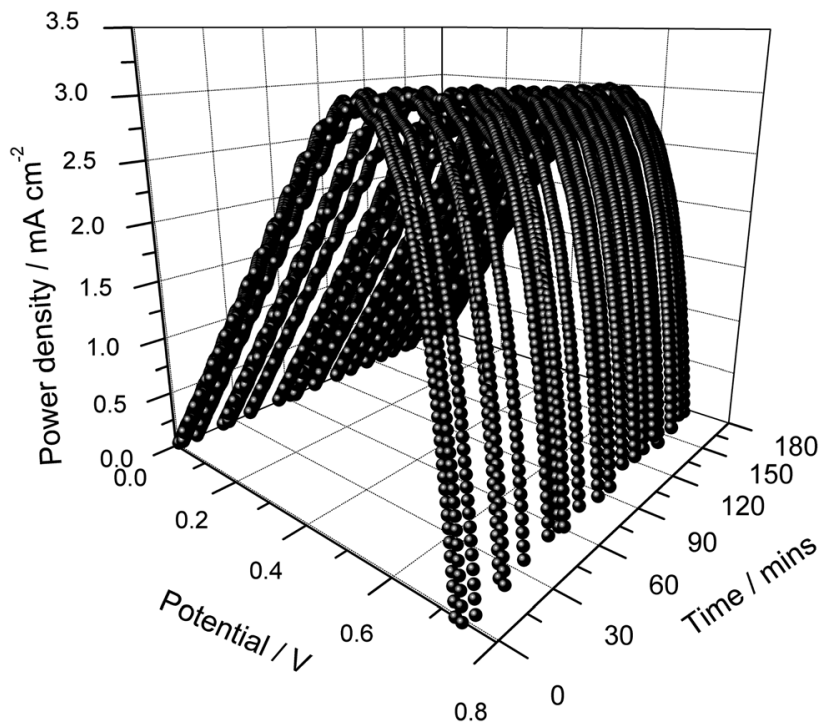

Fig. 3 Power density versus cell potential as a function of illumination time of the DSSC assembled using a single layer photo-electrode without frustules.

increasing temperature. ${ }^{42}$ Conversely, the open-circuit voltage $\left(V_{\text {oc }}\right)$ exhibits a small drop from 722 to $700 \mathrm{mV}$, which can also be a result of an increase of the charge transport kinetics with increasing temperature. ${ }^{\mathbf{4 1 , 4 3}}$ Therefore, when the cell is at open circuit, the increase of charge transport can increase the charge recombination kinetics (recombination of photo-generated electrons with tri-iodides in the electrolyte) shifting the Fermi level to more positive potentials, leading to a drop of $V_{\mathrm{oc}}{ }^{\mathbf{4 4 , 4 5}}$

The power density versus cell potential curves for different illumination periods are shown in Fig. 3. As seen in Fig. 2 and 3, the cells exhibit very good short-term stability owing to the use of an appropriate gel polymer electrolyte that suppresses the evaporation of liquid components. It is noteworthy that these cells are unsealed and assembled just by sandwiching a thin layer of the electrolyte between the photo-electrode and counter electrode.

The energy conversion efficiency of the cells dropped slightly from $\sim 3.1$ to $\sim 3.0 \%$ with time, which can be due to an increase of the recombination kinetics with increasing temperature. This is evidenced by the decrease of the fill factor with the increasing illumination period. The cell performances are very stable, and the variations in performance parameters are regarded as artifacts due to the temperature increase with the prolonged illumination. ${ }^{\mathbf{4 1 - 4 4}}$ This temperature effect was evident since the cells regained their original efficiency after a sufficient relaxation time ( $\sim 12 \mathrm{~h})$ (results are not shown). The average

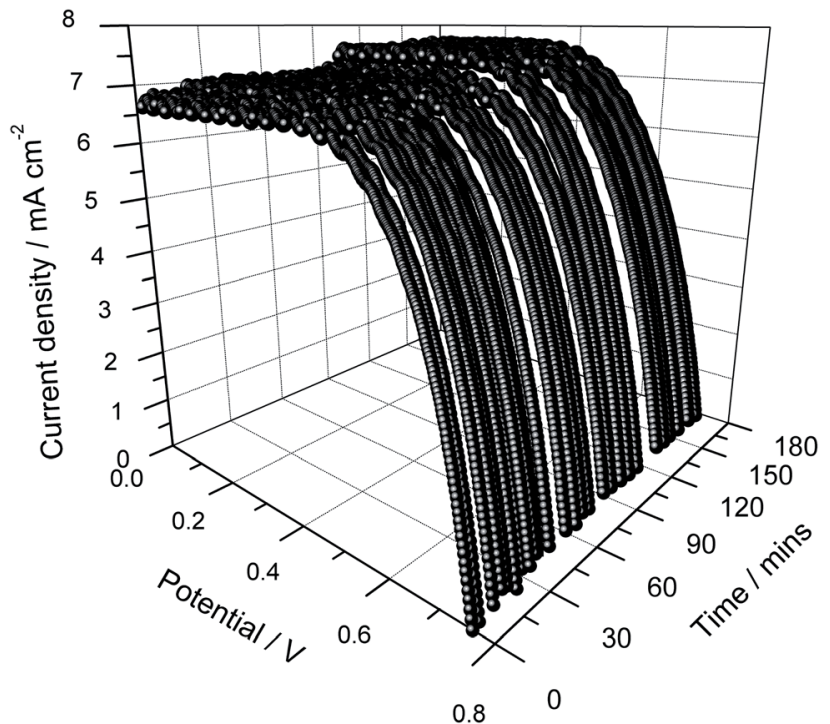

Fig. 4 Current density versus cell potential as a function of illumination time of the DSSC assembled using a double layer of the photoelectrode without frustules.

values of the solar cell performance parameters $J_{\mathrm{sc}}, V_{\mathrm{oc}}$, fill factor (ff) and efficiency $(\eta)$ for the $3 \mathrm{~h}$ duration are given Table 2 .

\subsection{DSSC with a double layer photoelectrode without frustules}

The $J-V$ curves of the quasi solid-state DSSC prepared using a double layer of the photo-electrode without frustules are shown in Fig. 4 as a function of illumination time. This doublelayered cell (Fig. 4) shows higher photo-currents compared to the single-layer cell (Fig. 2). In this case also, measurements were conducted for about 3 hours under continuous illumination. As shown in Fig. 4, within 3 hours, the $J_{\text {sc }}$ increased from 6.6 to $7.5 \mathrm{~mA} \mathrm{~cm}^{-2}$ while the open-circuit voltage $\left(V_{\mathrm{oc}}\right)$ showed a small drop, from 754 to $728 \mathrm{mV}$. As for the single-layer cells, this can be attributed to the small temperature increase during prolonged irradiation.

The power density versus cell potential curves are shown in Fig. 5 as a function of illumination time. This cell shows good stability though some minor fluctuations are visible. The energy conversion efficiency of the cell dropped slightly from $\sim 3.41$ to $\sim 3.36 \%$, possibly due to the heating effects resulting from the prolonged irradiation. Compared to the single-layer cell, this double layer cell showed cell performance enhancements of about 6.6, 2.9, and 9.9\% for $J_{\mathrm{sc}}, V_{\mathrm{oc}}$ and efficiency, respectively.

Table 2 Performance parameters of the DSSCs assembled with different layers

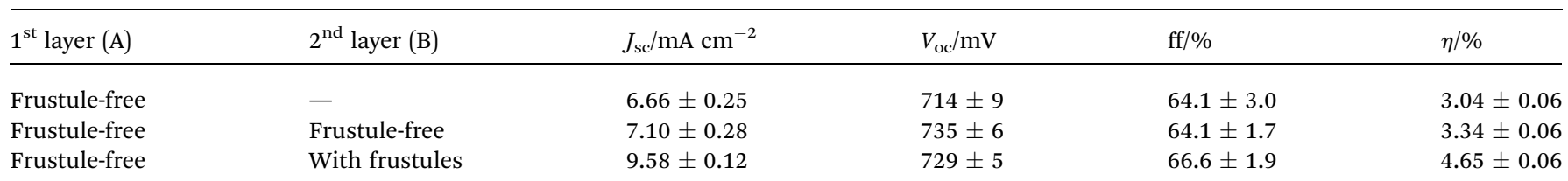




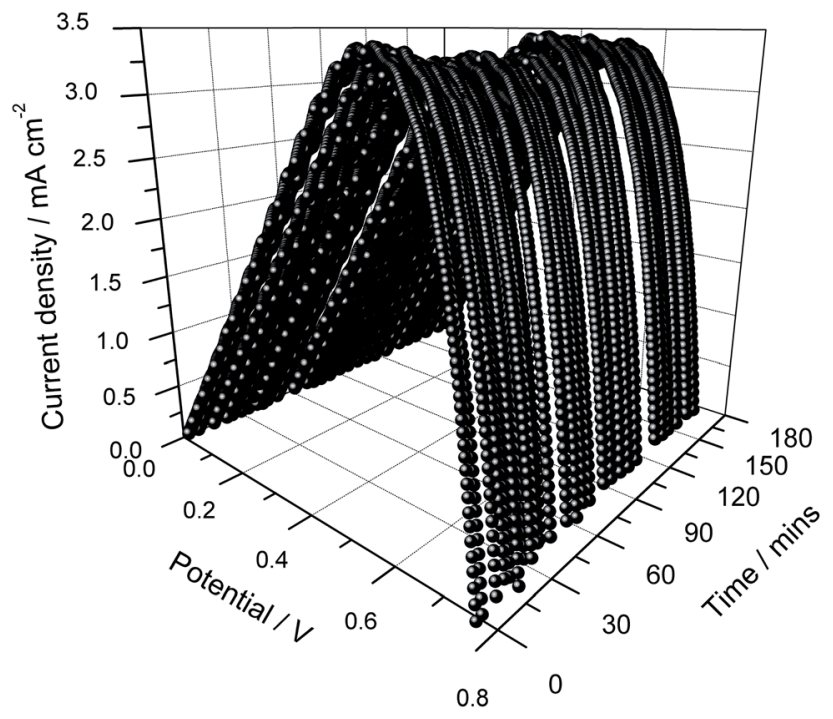

Fig. 5 Power density versus cell potential as a function of illumination time of the DSSC assembled using a double layer of the photo-electrode without frustules.

These enhancements are believed to be due to an increase of the adsorption of dye, which is the light-absorbing species in the photo-anode. The average values of the $J_{\mathrm{sc}}, V_{\mathrm{oc}}$, ff and efficiency in 3 hours are given in Table 2. The average fill factor remained unchanged for both types of cells.

\subsection{DSSC with a double layer photoelectrode with frustules} (top layer with and bottom layer without frustules)

The $J-V$ curves of the quasi solid-state DSSC prepared using a double layer photo-electrode with incorporated frustules are shown in Fig. 6 for about 3 hours of continuous illumination. Power density versus cell potential as a function of illumination time of the DSSC assembled using a photo-electrode containing

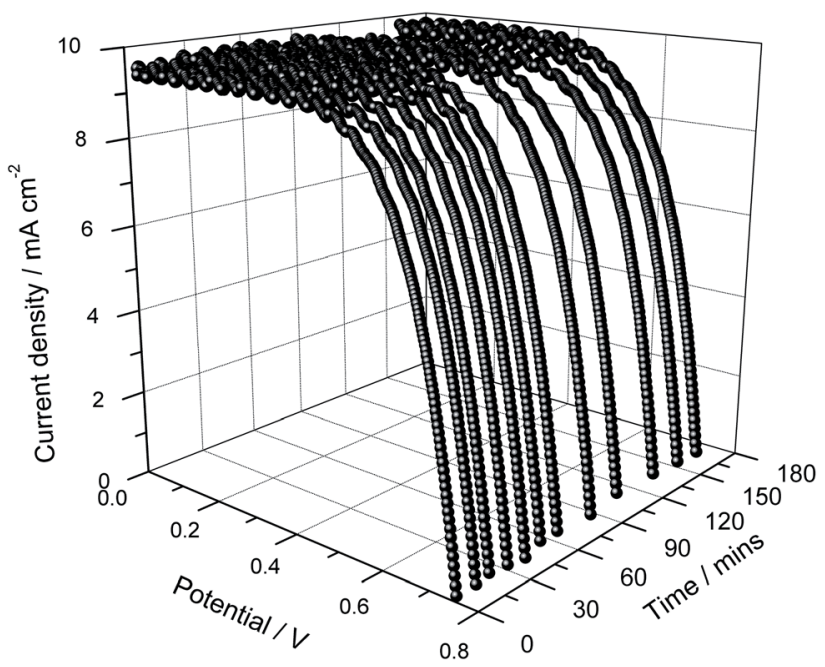

Fig. 6 Current density versus cell potential as a function of illumination time of the DSSC assembled using a double layer photo-electrode where one of the layers contains diatom frustules. double layers (one with and one without frustules) is shown in Fig. 7. The first layer contains no frustules, while the second layer of the photo-electrode contains small amounts of diatom frustules (mass fraction, frustule : $\mathrm{TiO}_{2}=1: 100$ ). The incorporated frustules are expected to increase the effective surface area of the photo-electrode compared to that of the photoelectrode prepared using only $\mathrm{TiO}_{2}$ nanoparticles due to the relatively large size of the frustules and their very porous nanostructure (Fig. 1). As seen in Fig. 6, the photo-current density has indeed improved significantly for the DSSC incorporated with frustules.

After 3 hours of illumination, the initial $J_{\mathrm{sc}}$ of 9.36 increased to $9.62 \mathrm{~mA} \mathrm{~cm}^{-2}$. Similar to the trend shown by the cells discussed above, the $V_{\text {oc }}$ decreased from 738 to $724 \mathrm{mV}$ with time. The efficiency also decreased from 4.72 to $4.58 \%$ with the increasing irradiation time as a result of the $V_{\text {oc }}$ drop. As in the earlier described cases, all these effects exhibited with illumination time are regarded as artifacts due to the increasing temperature, and the cells regained their efficiency after sufficient relaxation time.

The average values of the $J_{\mathrm{sc}}, V_{\mathrm{oc}}$ and efficiency are given in Table 2 for these DSSCs incorporated with diatom frustules. Highlighting the positive effect of incorporation of diatom frustules, this DSSC shows a remarkable improvement of 35, 4, and $39 \%$ for $J_{\mathrm{sc}}$, ff and efficiency compared to the frustule-free double-layered cells. However, the average $V_{\text {oc }}$ shows a slight drop of about $0.8 \%$. The efficiency and $J_{\text {sc }}$ enhancements exhibited due to the frustule containing $2^{\text {nd }}$ layer are remarkable. Compared to those of the single-layer DSSC, the efficiency and $J_{\mathrm{sc}}$ of the double layer DSSC with a second layer containing frustules improved by 53 and 44\%, respectively. In comparison, using a frustule-free second layer resulted in only 9.9 and $6.6 \%$ enhancements compared to the single-layer photoelectrode.

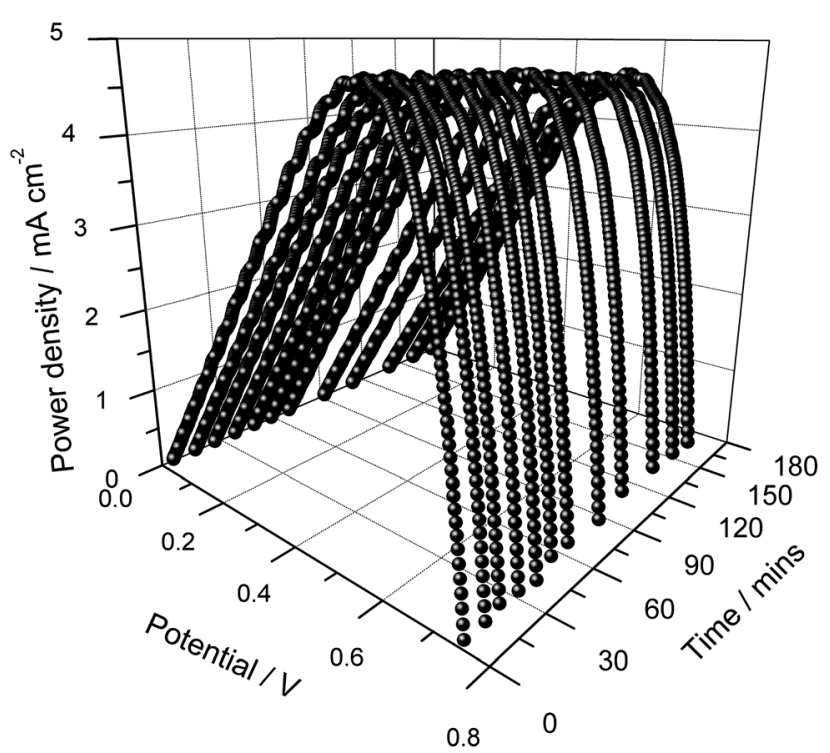

Fig. 7 Power density versus cell potential as a function of illumination time of the DSSC assembled using a photo-electrode containing double layers, one with and one without frustules. 


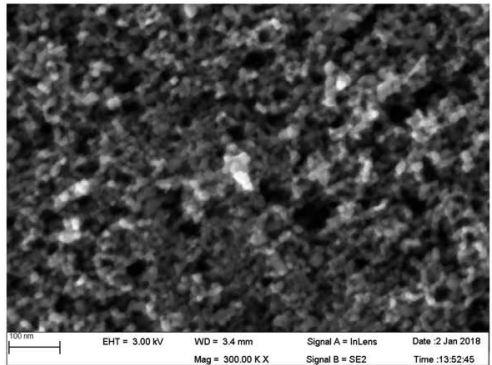

(a)

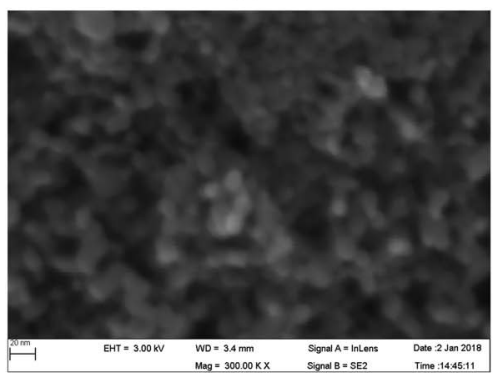

(b)

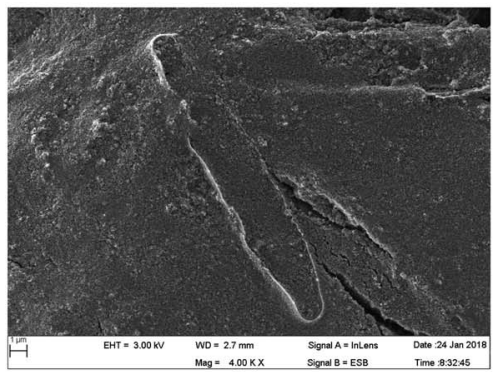

(c)

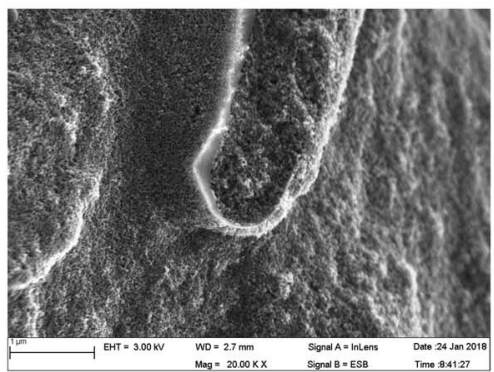

(d)

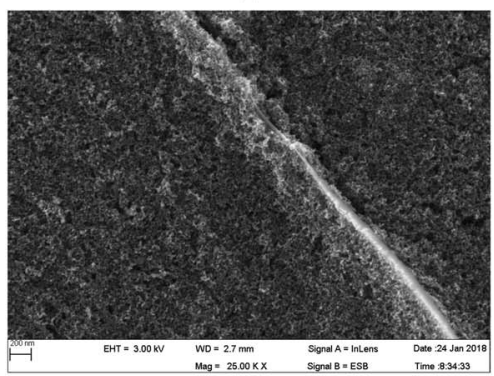

(e)

Fig. 8 SEM image of double-layer photo-electrodes prepared without frustules ( $a$ and $b$ ) and with frustules $(c-e)$ in the top layer. The bottom layer is a frustule-free $\mathrm{TiO}_{2}$ nanoparticle coating.
Therefore, the incorporation of frustules in the $2^{\text {nd }}$ layer is a promising way to increase the DSSC performances significantly.

\subsection{SEM analysis}

For a better understanding of the structural and morphological changes, the SEM images of different $\mathrm{TiO}_{2}$ layers are shown in Fig. 8. In addition, a schematic illustration of the different layers of $\mathrm{TiO}_{2}$ studied is given in Fig. 9.

The SEM images of the 1st layer prepared without frustules are shown in Fig. 8(a) and (b). It is clear that there are no cracks or pinholes in this film. The structure is highly porous, and the layer is composed of $\mathrm{TiO}_{2}$ crystallites with an average size of $14 \mathrm{~nm}$. The size of the observed crystallites is thus in agreement with the stated particle size of the P90 powder (supplier specification). When two such layers are present, the structure does not change, only the thickness increases. Therefore, the SEM images are not shown for the frustule-free double layer.

The SEM image of the photo-electrode prepared with the double layer (top layer with and bottom layer without frustules), is shown in Fig. 8(c-e) at different magnifications. Traces of diatom frustules well covered by $\mathrm{TiO}_{2}$ nanoparticles $(\sim 14 \mathrm{~nm})$ are clearly visible in the image (Fig. 8(c-e)). Since the frustulecontaining layer is prepared on top of a layer of $\mathrm{TiO}_{2}$ nanoparticles, the frustules are covered well from all directions, as seen in Fig. 8(c). Enlarged SEM images that trace small uncovered edges of the frustules are shown in Fig. 8(c-e). The light enters even though these uncovered regions and can undergo multiple reflections contributing to the enhancement of cell efficiency. Some cracks are visible in these frustule-containing layers; however, these cracks would not have an adverse effect on cell efficiency since the frustule-containing layer is prepared on a crack-free $\mathrm{TiO}_{2}$ layer. Thus, these cracks cannot contribute to internal short circuit, which deteriorates the cell performance. Conversely, these cracks can actually increase cell performance in this situation since they can improve dye adsorption.

A schematic illustration of the different layers of $\mathrm{TiO}_{2}$ used in this study is given in Fig. 9. The light rays that enter into the frustules will undergo multiple reflections, enhancing the possibility of their capture by dye molecules attached to the $\mathrm{TiO}_{2}$ nanoparticles. Preliminary studies actually revealed that the energy conversion efficiency of frustule-containing singlelayered DSSCs is less than that of single-layered frustule-free cells. Therefore, the addition of frustules contributed to a drop in the efficiency in single-layered photo-electrode based cells. This can be understood by studying the influence of the added frustules on electron transport. In order to obtain high efficiency, the injected electrons from the excited dye should be transported efficiently across the $\mathrm{TiO}_{2}$ film. The electron percolation through the single-layer electrodes without frustules is illustrated in Fig. 9(a) where the arrows indicate electron percolation. Though added nanostructured frustules can contribute to enhancing the light absorption of the photoelectrode, they cause a decrease in the electron transport through the photo-electrode, as shown for many other 


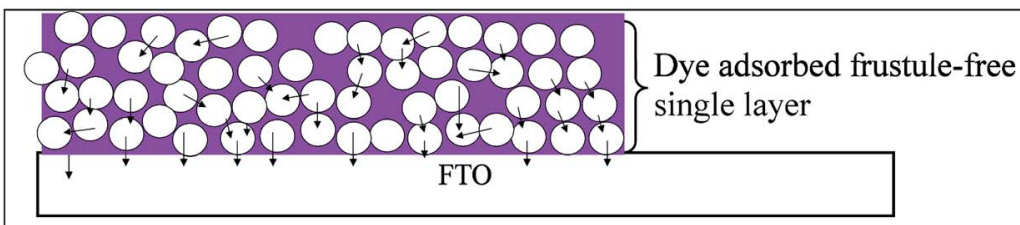

(a)

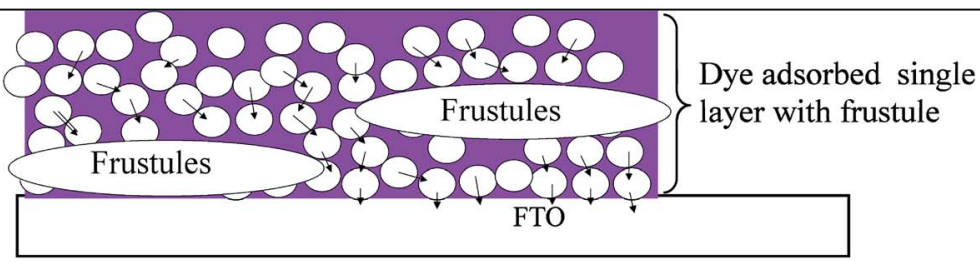

(b)

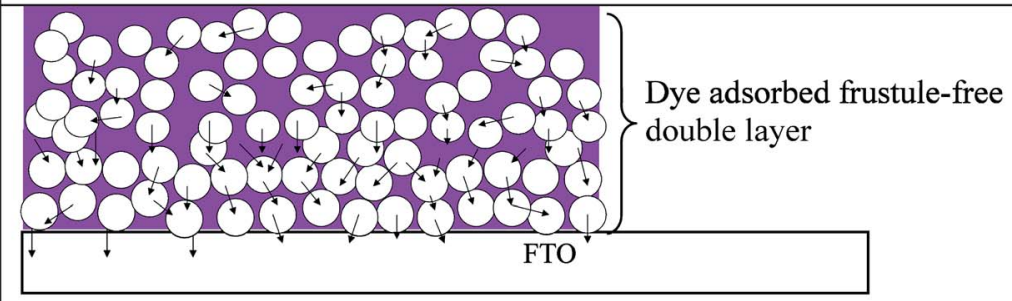

(c)

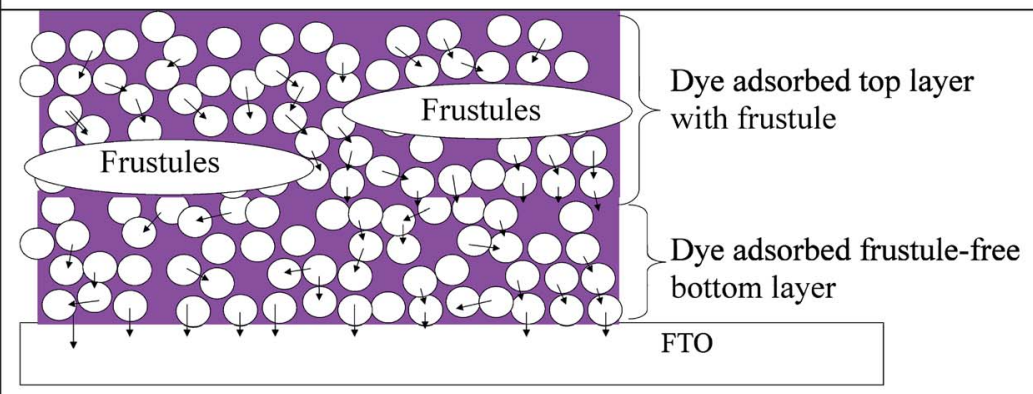

(d) (a) Electron transport is higher,

however, low efficiency due to

less dye adsorption due to

small thickness,

(b) Electron transport is poor,

however, dye adoption and

light scattering improve

(c) Electron transport and dye

adoption are higher, however,

light scattering is poor.

(d) Electron transport dye

adoption and light scattering

are higher

Fig. 9 Schematic illustration of photo-electrodes prepared with (a) a frustule-free single layer, (b) a single layer with frustules, (c) a frustule-free double layer and (d) a double layer with frustules inserted on top of a frustule-free compact layer (not to scale).

nanostructures added to DSSCs. ${ }^{\mathbf{1 2 , 1 4 , 1 7 - 1 9 , 4 6}}$ The observed efficiency drop of the frustule-containing single layer cell can, thus, result from a drop in electron conduction through the photoelectrode, as illustrated in Fig. 9(b), since frustules are made of inert silica that does not contribute to electron transport. The efficiency of the electrode containing a frustule-free double layer is moderate since the light scattering is poor, though dye adsorption and electron transport are high. However, a higher efficiency can be expected for the photo-electrode containing a layer with frustules on top of a frustule-free layer, as illustrated in Fig. 9(d). The dye adsorption, light scattering, and electron percolation are higher for this electrode compared to the other electrodes studied. This expected behavior of cell performance is confirmed by the average DSSC performance parameters given in Table 2 which are calculated from the current-voltage characteristic curves. The SEM images given in Fig. 8 also confirm the structure schematically illustrated in Fig. 9.

The performance (efficiency) of DSSCs with respect to irradiation time for both systems with and without diatom frustules is shown in Fig. 10. In addition, the efficiency of the single 


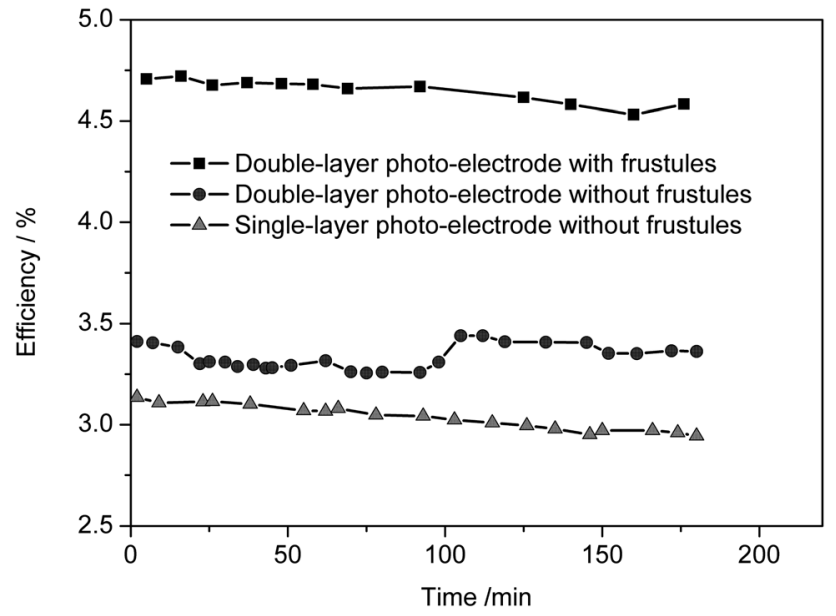

Fig. 10 The efficiency of DSSCs with respect to irradiation time for systems with and without diatom frustules. In addition, the efficiency of a frustule free single-layer cell is also shown.

layered cell is also shown in Fig. 10. The reported efficiency of $4.7 \%$ and $J_{\mathrm{sc}}$ of $9.64 \mathrm{~mA} \mathrm{~cm}{ }^{-2}$ are very good values for a quasisolid state DSSC that does not contain volatile solvents in the electrolyte. This work very clearly shows that the efficiency enhancement is due to the waveguiding and photon localization effects of incorporated frustules. Finally, it can be concluded that the efficiency in the double-layered quasi-sold state DSSC could be enhanced by $39 \%$ (from 3.34 to $4.65 \%$ ) by incorporating nanostructured diatom frustules into the $2^{\text {nd }}$ layer of the photo-electrode.

\section{Conclusions}

Quasi solid-state DSSCs containing diatom frustules were realized and studied in order to capitalize on the light trapping properties of complex 3-dimensional nanostructures designed by nature for scattering and trapping light for photosynthesis. The present study shows a promising approach to prepare $\mathrm{TiO}_{2}$ nanoparticle-based photo-electrodes, incorporating biologically formed silica nanostructures in order to take advantage of their waveguiding and photon localization effects to enhance light harvesting without impairing electron conduction. Single and double-layered photo-electrodes were prepared with various conformations of frustules and $\mathrm{TiO}_{2}$ nanocrystals on transparent conductive oxide substrates. SEM images revealed that a remarkable surface coverage of evenly distributed frustules and $\mathrm{TiO}_{2}$ nanoparticles could be achieved. For the cell construction, a gel polymer electrolyte without any volatile solvents was prepared to avoid physical or chemical instability of the cells. Stable cell performances were observed under continuous illumination for about 3 hours; minor variations observed during the illumination time are believed to be due to heating effects since the cells regained their original efficiencies after a sufficient relaxation time of about $12 \mathrm{~h}$.

For single layered photo-electrodes, the addition of frustules resulted in a drop in the efficiency, which may be caused by poor electron transport due to the added frustules despite the improved light harvesting. However, the double layer photo- electrode, prepared by adding $1 \%$ wt of frustules to the $2^{\text {nd }}$ layer, showed average $J_{\mathrm{sc}}, V_{\mathrm{oc}}$ and efficiency values of $9.58 \pm 0.12$ $\mathrm{mA} \mathrm{cm}{ }^{-2}, 729 \pm 5 \mathrm{mV}$ and $4.65 \pm 0.06$ under $1000 \mathrm{~W} \mathrm{~m}^{-2}$ irradiation.

This study clearly shows impressive efficiency and short circuit current density enhancements of $35 \%$ and $39 \%$, respectively, due to the incorporation of naturally built nanostructures extracted from ubiquitous species: diatom frustules. To the best of our knowledge, the present work reports the first quasi solid-state DSSC based on a photo-electrode with incorporated bio-formed nanostructures. This study thus confirmed that the light localization of diatom frustules can be used to enhance the efficiency of dye-sensitized solar cells.

\section{Conflicts of interest}

The Swedish Algae Factory received funding from the Swedish Energy Agency and then engaged Chalmers and the University of Gothenburg to perform an independent study.

\section{Acknowledgements}

The authors wish to acknowledge the research support provided by the Swedish Research Council (Grant number: 348-20144284), the Swedish Algae Factory, and the Swedish Energy Agency (Grant number: 43227-1).

\section{References}

1 J. S. Shaikh, N. S. Shaikh, S. S. Mali, J. V. Patil, K. K. Pawar, P. Kanjanaboos and P. S. Patil, Nanoarchitectures in dyesensitized solar cells: metal oxides, oxide perovskites and carbon-based materials, Nanoscale, 2018, 10(11), 4987-5034.

2 T. M. W. J. Bandara, W. J. M. J. S. R. Jayasundara, H. D. N. S. Fernado, M. A. K. L. Dissanayake, L. A. A. De Silva, I. Albinsson, M. Furlani and B. E. Mellander, Efficiency of $10 \%$ for quasi-solid state dye-sensitized solar cells under low light irradiance, J. Appl. Electrochem., 2015, 45(4), 289-298.

3 A. N. Filippin, J. R. Sanchez-Valencia, J. Idígoras, T. C. Rojas, A. Barranco, J. A. Anta and A. Borras, Plasma assisted deposition of single and multistacked $\mathrm{TiO}_{2}$ hierarchical nanotube photoanodes, Nanoscale, 2017, 9(24), 8133-8141.

4 K. Sato, M. Dutta and N. Fukata, Inorganic/organic hybrid solar cells: optimal carrier transport in vertically aligned silicon nanowire arrays, Nanoscale, 2014, 6(11), 6092-6101.

5 Y. Wei, C. Xu, S. Xu, C. Li, W. Wu and Z. L. Wang, Planar Waveguide-Nanowire Integrated Three-Dimensional DyeSensitized Solar Cells, Nano Lett., 2010, 10(6), 2092-2096.

6 X. Zhang, S. Yang, H. Zhou, J. Liang, H. Liu, H. Xia, X. Zhu, Y. Jiang, Q. Zhang, W. Hu and X. Zhuang, Perovskite-Erbium Silicate Nanosheet Hybrid Waveguide Photodetectors at the Near-Infrared Telecommunication Band, Adv. Mater., 2017, 29(21), 1604431.

7 W. Q. Wu, Y. F. Xu, H. S. Rao, H. L. Feng, C. Y. Su and D. B. Kuang, Constructing 3D branched nanowire coated macroporous metal oxide electrodes with homogeneous or 
heterogeneous compositions for efficient solar cells, Angew. Chem., Int. Ed., 2014, 53(19), 4816-4821.

8 M. Grätzel, Recent advances in sensitized mesoscopic solar cells, Acc. Chem. Res., 2009, 42(11), 1788-1798.

9 D. Chen, F. Huang, Y. B. Cheng and R. A. Caruso, Mesoporous anatase $\mathrm{TiO}_{2}$ beads with high surface areas and controllable pore sizes: a superior candidate for high-performance dye-sensitized solar cells, Adv. Mater., 2009, 21(21), 2206-2210.

10 Y. Chiba, A. Islam, Y. Watanabe, R. Komiya, N. Koide and L. Y. Han, Jpn. J. Appl. Phys., Part 2, 2006, 45, L638.

11 S. Ito, P. Chen, P. Comte, M. K. Nazeeruddin, P. Liska, P. Péchy and M. Grätzel, Fabrication of screen-printing pastes from $\mathrm{TiO}_{2}$ powders for dye-sensitized solar cells, Prog. Photovoltaics, 2007, 15(7), 603-612.

12 M. Yu, Y. Z. Long, B. Sun and Z. Fan, Recent advances in solar cells based on one-dimensional nanostructure arrays, Nanoscale, 2012, 4(9), 2783-2796.

13 N. M. Ali and N. H. Rafat, Modeling and simulation of nanorods photovoltaic solar cells: A review, Renewable Sustainable Energy Rev., 2017, 68, 212-220.

14 H. Sun, J. Deng, L. Qiu, X. Fang and H. Peng, Recent progress in solar cells based on one-dimensional nanomaterials, Energy Environ. Sci., 2015, 8(4), 1139-1159.

15 Y. Chiba, A. Islam, Y. Watanabe, R. Komiya, N. Koide and L. Han, Dye sensitized solar cells with conversion efficiency of 11.1\%, Jpn. J. Appl. Phys., 2006, 45, 24-28.

16 S. Ito, T. N. Murakami, P. Comte, P. Liska, C. Grätzel, M. K. Nazeeruddin and M. Grätzel, Fabrication of thin film dye sensitized solar cells with solar to electric power conversion efficiency over 10\%, Thin Solid Films, 2008, 516, 4613-4619.

17 J. Ding, Y. Zhao, J. Duan, Y. Duan and Q. Tang, Hollow optical fiber induced solar cells with optical energy storage and conversion, Chem. Commun., 2017, 53(90), 12233-12235.

18 S. Manouchehri, J. Zahmatkesh and M. H. Yousefi, Twodimensional optical fiber-based dye-sensitized solar cell simulation: the effect of different electrodes and dyes, $J$. Comput. Electron., 2018, 17(1), 329-336.

19 A. Mihi, C. Zhang and P. V. Braun, Transfer of Preformed Three-Dimensional Photonic Crystals onto Dye-Sensitized Solar Cells, Angew. Chem., Int. Ed., 2011, 50(25), 5712-5715.

20 Y. Li, M. E. Calvo and H. MÍguez, Light Harvesting Enhancement in Photonic-Crystal-Integrated Flexible DyeSensitized Solar Cell, in Optical Nanostructures and Advanced Materials for Photovoltaics, Optical Society of America, 2015, November, pp. JM2C-5.

21 A. Polman and H. A. Atwater, Photonic design principles for ultrahigh-efficiency photovoltaics, Nat. Mater., 2012, 11, 174-177.

22 P. Bermel, C. Luo, L. Zeng, L. C. Kimerling and J. D. Joannopoulos, Improving thin-film crystalline silicon solar cell efficiencies with photonic crystals, Opt. Express, 2007, 15, 16986-17000.

23 S. N. Olof, J. A. Grieve, D. B. Phillips, H. Rosenkranz, M. L. Yallop, M. J. Miles, A. J. Patil, S. Mann and
D. M. Carberry, Measuring nanoscale forces with living probes, Nano Lett., 2012, 12(11), 6018-6023.

24 X. Chen, C. Wang, E. Baker and C. Sun, Numerical and experimental investigation of light trapping effect of nanostructured diatom frustules, Sci. Rep., 2015, 5, 11977.

25 J. Romann, J. C. Valmalette, M. S. Chauton, G. Tranell, M. A. Einarsrud and O. Vadstein, Wavelength and orientation dependent capture of light by diatom frustule nanostructures, Sci. Rep., 2015, 5, 17403.

26 F. E. Round, R. M. Crawford and D. G. Mann, The Diatoms, Cambridge University Press, 1990.

27 P. Tréguer, C. Bowler, B. Moriceau, S. Dutkiewicz, M. Gehlen, O. Aumont, L. Bittner, R. Dugdale, Z. Finkel, D. Iudicone and O. Jahn, Influence of diatom diversity on the ocean biological carbon pump, Nat. Geosci., 2018, 11(1), 27.

28 N. Kröger and N. Poulsen, Diatoms-from cell wall biogenesis to nanotechnology, Annu. Rev. Genet., 2008, 42, 83-107.

29 A. K. Noren, Characterization of Structure and Optical Properties of Diatoms for Improved Solar Cell Efficiency, Master's Thesis, Norwegian University of Science and Technology, 2011.

30 E. De Tommasi, I. Rea, L. De Stefano, P. Dardano, G. Di Caprio, M. A. Ferrara and G. Coppola, Optics with diatoms: towards efficient, bioinspired photonic devices at the micro-scale, in Optical Methods for Inspection, Characterization, and Imaging of Biomaterials, International Society for Optics and Photonics, 2013, vol. 8792, p. 879200.

31 G. Di Caprio, G. Coppola, L. D. Stefano, M. D. Stefano, A. Antonucci, R. Congestri and E. D. Tommasi, Shedding light on diatom photonics by means of digital holography, J. Biophotonics, 2014, 7(5), 341-350.

32 D. P. Medarević, D. Lošić and S. R. Ibrić, Diatoms-nature materials with great potential for bioapplications, Chem. Ind., 2016, 70(6), 613-627.

33 H. E. Townley, K. L. Woon, F. P. Payne, H. White-Cooper and A. R. Parker, Modification of the physical and optical properties of the frustule of the diatom Coscinodiscus wailesii by nickel sulfate, Nanotechnology, 2007, 18(29), 295101.

34 J. Toster, K. S. Iyer, W. Xiang, F. Rosei, L. Spiccia and C. L. Raston, Diatom frustules as light traps enhance DSSC efficiency, Nanoscale, 2013, 5(3), 873-876.

35 J. K. Wang and M. Seibert, Prospects for commercial production of diatoms, Biotechnol. Biofuels, 2017, 10(1), 16.

$36 \mathrm{~J}$. Campbell and G. Rorrer, Integration of biological photonic crystals in dye-sensitized solar cells for enhanced photocurrent generation, in Nanobiosystems: Processing, Characterization, and Applications VI, International Society for Optics and Photonics, 2013, October, vol. 8817, p. 88170Q.

37 D.-R. Huang, Y.-J. Jiang, R.-L. Liou, C.-H. Chen, Y.-A. Chen and C. H. Tsai, Enhancing the efficiency of dye-sensitized solar cells by adding diatom frustules into $\mathrm{TiO}_{2}$ working electrodes, Appl. Surf. Sci., 2015, 347, 64-72.

38 K. Kakiage, Y. Aoyama, T. Yano, K. Oya, J. Fujisawa and M. Hanaya, Highly-efficient dye-sensitized solar cells with 
collaborative sensitization by silyl-anchor and carboxyanchor dyes, Chem. Commun., 2015, 51, 15894-15897.

39 V. Sugathan, E. John and K. Sudhakar, Recent improvements in dye sensitized solar cells: A review, Renewable Sustainable Energy Rev., 2015, 52, 54-64.

40 M. S. Su'ait, M. Y. A. Rahman and A. Ahmad, Review on polymer electrolyte in dye-sensitized solar cells (DSSCs), Sol. Energy, 2015, 115, 452-470.

41 T. M. W. J. Bandara, W. J. M. J. S. R. Jayasundara, M. A. K. L. Dissanayake, H. D. N. S. Fernando, M. Furlani, I. Albinsson and B. E. Mellander, Quasi solid state polymer electrolyte with binary iodide salts for photoelectrochemical solar cells, Int. J. Hydrogen Energy, 2014, 39(6), 2997-3004.

42 U. Mehmood, A. Al-Ahmed, F. A. Al-Sulaiman, M. I. Malik, F. Shehzad and A. U. H. Khan, Effect of temperature on the photovoltaic performance and stability of solid-state dye-sensitized solar cells: A review, Renewable Sustainable Energy Rev., 2017, 79, 946-959.
43 R. Jiang and G. Boschloo, The Effect of Illumination Direction and Temperature on Dye-Sensitized Solar Cells with Viscous Cobalt Complex-Based Electrolytes, Inorganics, 2018, 6(2), 60.

44 J. Bisquert, A. Zaban, M. Greenshtein and I. Mora-Seró, Determination of rate constants for charge transfer and the distribution of semiconductor and electrolyte electronic energy levels in dye-sensitized solar cells by open-circuit photovoltage decay method, J. Am. Chem. Soc., 2004, 126(41), 13550-13559.

45 J. Gong, K. Sumathy, Q. Qiao and Z. Zhou, Review on dyesensitized solar cells (DSSCs): advanced techniques and research trends, Renewable Sustainable Energy Rev., 2017, 68, 234-246.

46 M. Ye, X. Wen, M. Wang, J. Iocozzia, N. Zhang, C. Lin and Z. Lin, Recent advances in dye-sensitized solar cells: from photoanodes, sensitizers and electrolytes to counter electrodes, Mater. Today, 2015, 18(3), 155-162. 